\title{
Stability of E. Reyssner's plates on the elastic non-winkler foundation
}

\author{
Serdar Yazyev ${ }^{1, *}$, Dmitriy Vysokovsky ${ }^{1}$, and Polina Kulinich $^{1}$ \\ ${ }^{1}$ Don State Technical University, pl. Gagarina, 1, Rostov-on-Don, 344010, Russia
}

\begin{abstract}
The problem of E. Reyssner's plate stability lying on an elastic three-dimensional layer with desired elastic constants. The end surfaces of layer are smooth, connection holding. It is assumed that the plate is in a flat stress-strain state of the effects on its cylindrical surface of the self-balanced load, with some numerical parameter characterizing the magnitude of the load at loss of stability of the plate. From the conditions of restraint ties one obtain a system of equations for determining the numerical parameter. Method is given for calculating the lowest value of the parameter at which the plate's loss of stability is fixed. As special cases, the results of the classical theory and model of Winkler foundation are present.
\end{abstract}

\section{Introduction}

Let us consider the plate having the shape of $\Omega$ and limited by cylindrical contour $\Gamma$ lying on the elastic layer of the three-dimensional: $-\infty(x, y)<\infty ; 0 \leq z_{1} \leq h_{1}$. Let the elastic constants be $E_{l}, v_{l}$ layer, plate thickness h, its elastic constants $E, v, D=h^{3} E / 12\left(1-v^{2}\right)$. The end surfaces of layer are smooth, connection holding. Let us assume that the plate is in a flat stress-strain state of the effects on the contour $\Gamma$ of self-balanced load $\lambda P_{n}(s), \lambda P_{\tau}(s)$, where $\lambda$ - some numerical parameter, characterizing the magnitude of the load at loss of stability of the plate. From these conditions,

$$
\begin{gathered}
\left.\sigma_{n}\right|_{\Gamma}=\lambda P_{n}(S),\left.\tau_{n s}\right|_{\Gamma}=-\lambda P_{\tau}(S),\left.\tau_{\alpha Z}\right|_{Z= \pm h / 2}=0,\left.\sigma_{Z}\right|_{Z=-h / 2}=0 ; \\
\left.\tau_{\alpha Z_{1}}\right|_{Z_{1}=0 ; h_{1}}=0,\left.\quad \omega_{1}\right|_{Z_{1}=0}=0,(\alpha=x, y) ; \\
\omega=\left.\omega_{1}\right|_{Z_{1}=h_{1}},\left.\quad \sigma_{Z}\right|_{Z=h / 2}=\left.\sigma_{Z_{1}}\right|_{Z_{1}=h_{1}} .
\end{gathered}
$$

\section{Problem definition}

Suppose that for some reason, plate bent a bit. Consider the following conditions of bifurcation of forms of compressed slabs according to Euler equilibrium.

\footnotetext{
*Corresponding author: serdariaziev@gmail.com
} 
If the plate is loaded by power system (1), when of its bending compressive forces give the component in the same direction as the transverse load:

$$
q=(x, y)=\lambda\left(\omega_{x x} F_{y y}+\omega_{y y} F_{x x}-2 \omega_{x y} F_{x y}\right)
$$

Where $\Phi(x, y)$ - function of longitudinal forces in the plate, determined by solving biharmonic problem [1]. The relations between the power and the geometric characteristics of the plate of E. Reysner have the form

$$
\begin{gathered}
M_{\alpha}=-D\left(\partial_{\alpha}^{2}+v \partial_{\beta}^{2}\right) \omega+0,1 h^{2} \partial_{\alpha} Q_{\alpha}+0,1 h^{2} v /(1-v)\left(\partial_{1} Q_{x}+\partial_{2} Q_{y}\right)-\frac{h^{2} v}{12(1-v)} q(x, y) \\
(\alpha=x, y) ; \\
M_{x y}=-D(1-v) \partial_{1} \partial_{2} \omega+0,1 h^{2}\left(\partial_{2} Q_{x}+\partial_{1} Q_{y}\right) ; \\
\partial_{\alpha} M_{\alpha}-\partial_{\beta} M_{\alpha \beta}=Q_{\alpha}, \quad(\alpha=x, y), \quad(\beta=y, x) \\
\left(0,1 h^{2} D^{2}-1\right) Q_{n}+0,1 h^{2} \frac{1}{1-v} \partial_{\alpha}\left(\partial_{1} Q_{x}+\partial_{2} Q_{y}\right)=D \alpha D^{2} \omega+\frac{h^{2}}{12} \frac{v}{1-v} \partial_{\alpha} q(x, y) \\
(\alpha=x, y) ;
\end{gathered}
$$

Stress-strain state of three-dimensional elastic layer is described by the solution of homogeneous equations of the theory of elasticity in displacements [2]:

$$
\begin{gathered}
|| \begin{array}{l}
u \\
v
\end{array}||=\cos z_{1} D_{1}|| \begin{array}{l}
u_{0} \\
v_{0}
\end{array}||-\frac{x_{1}}{2} \frac{z_{1} \sin z_{1} D}{D} \partial_{\alpha} \theta_{0} ; \\
\omega_{1}=\frac{\sin z_{1} D_{1}}{D} \omega_{0}^{\prime}+\frac{x_{1}}{2}\left(\frac{\sin z_{1} D}{D}-z_{1} \cos z_{1} D\right) \theta_{0} ; \\
\frac{1}{\mu_{1}} \tau_{\alpha Z_{1}}=-\frac{\sin z_{1} D}{D}\left(D^{2}|| \begin{array}{l}
u_{0} \\
v_{0}
\end{array}||-\partial_{\alpha} \omega_{0}^{\prime}-x_{1} z_{1} \cos z_{1} D \partial_{\alpha} \theta_{0}\right) ; \\
\frac{1}{\mu_{1}} \tau_{z_{1}}=2 \cos z_{1} D_{1} \omega_{0}^{\prime}+\left[x_{1} z_{1} D \sin z_{1} D+\left(x_{1}-1\right) \cos z_{1} D\right] \theta_{0} .
\end{gathered}
$$

Here $z_{1} \in\left[0 ; h_{1}\right], u_{0}(x, y), v_{0}(x, y), \omega_{0}^{\prime}(x, y), \theta_{0}=\partial_{1} u_{0}+\partial_{2} v_{0}+\omega_{0}^{\prime}$ - basic unknown functions. Expressions (10) (11) identically satisfy the conditions (2). Other conditions on the ends of the plate and layer allow to solve the problem of the of bifurcation forms of balance of system and to determine the value of the parameter $\lambda$. For this purpose substitute $(10)-(12)(2)$ and (3) [3-6]. Get

$$
\frac{\sin z_{1} D}{D} D^{2}|| \begin{aligned}
& u_{0} \\
& v_{0}
\end{aligned} \|-\partial_{\alpha} \omega_{0}^{\prime}+x_{1} z_{1} \cos z_{1} D \times \partial_{\alpha} \theta_{0}=0 ;
$$




$$
\begin{gathered}
\omega=\frac{\sin z_{1} D}{D} \omega_{0}^{\prime}+\frac{x_{1}}{2}\left(\frac{\sin z_{1} D}{D}-h_{1} \cos h_{1} D\right) \theta_{0} ; \\
\partial_{1} Q_{x}+\partial_{2} Q_{y}+q(x, y)=\mu_{1} \times \\
\times\left[x_{1} h_{1} D \sin h_{1} D \theta_{0}+\left(x_{1}-1\right) \cos h_{1} D \theta_{0}+2 \cos h_{1} D \omega_{0}^{\prime}\right]
\end{gathered}
$$

Thus, the general solution of the problem of stress-strain state "plate - a layer" system is reduced to solving the system of Equations 13-15 [7-8]. Let us transform it. From (8) and (15) we have

$$
\begin{aligned}
D D^{4} \omega & =\left(1-\frac{h^{2}}{60} \frac{12-v}{1-v} D^{2}\right) q(x, y)-\mu_{1}\left(1-0,1 h^{2} \frac{2-v}{1-v} D^{2}\right) \times \\
& \times\left\{2 \cos h_{1} D \omega_{0}^{\prime}+\left[x_{1} h_{1} D \sin h_{1}+\left(x_{1}-1\right) \cos h_{1} D\right] \theta_{0}\right\} .
\end{aligned}
$$

Substituting (14) into (16) leads to the Equation

$$
\begin{gathered}
{\left[D D^{3} \sin h_{1} D+2 \mu_{1}\left(1-0,1 h^{2} \frac{2-v}{1-v} D^{2}\right) \cos h_{1} D\right] \omega_{0}+} \\
+\left\{D \frac{x_{1}}{2}\left(\frac{\sin z_{1} D}{D}-h_{1} \cos h_{1} D\right)+\mu_{1}\left(1-0,1 h^{2} \frac{2-v}{1-v} D^{2}\right) \times\right. \\
\left.\times\left[x_{1} h_{1} D \sin h_{1} D+\left(x_{1}-1\right) \cos h_{1} D\right] \theta_{0}\right\}=\left(1-\frac{h^{2}}{60} \frac{12-v}{1-v} D^{2}\right) q(x, y)
\end{gathered}
$$

Next, the whole issue is to solve the system of Equations 13 and 17 which can be built by semi-inverse methods. For this we represent the desired solution as the sum of two independent solutions - potential and vortex. It is easy to show that the vortex solution does not solve the problem of stability of the system "plate - layer", and therefore we"ll focus only on potential solutions [9]. Let

$$
u_{0}=L_{1} \partial_{1} \Pi, v_{0}=L_{1} \partial_{2} \Pi, \omega_{0}^{\prime}=L_{2} D^{2} \Pi,
$$

where $L_{l}$ и $L_{2}$ - some differential operators; $P$ - stress function. Substituting (18) in (13), we obtain the equation

$$
\left[\left(D \sin h_{1} D+x_{1} h_{1} D^{2} \cos h_{1} D\right) L_{1}-\left(D \sin h_{1} D-x_{1} h_{1} D^{2} \cos h_{1} D\right) L_{2}\right] \Pi=0,
$$

which are satisfied with identically for the obvious choice of operators:

$$
L_{i}=\frac{\sin h_{1} D}{D}+(-1)^{i} x_{1} h_{1} \cos h_{1} D
$$

The unknown functions take the form

$$
\| \begin{aligned}
& u_{0} \\
& v_{0}
\end{aligned}||=\left(\frac{\sin h_{1} D}{D}-x_{1} h_{1} \cos h_{1} D\right)|| \begin{aligned}
& \partial_{1} \\
& \partial_{2}
\end{aligned}|| \Pi
$$




$$
\begin{gathered}
\omega_{0}^{\prime}=\left(\frac{\sin h_{1} D}{D}+x_{1} h_{1} \cos h_{1} D\right) D^{2} \Pi ; \\
\theta_{0}=2 D \sin h_{1} D \Pi .
\end{gathered}
$$

The expressions for the bending plate we find from expression (14)[10-11]:

$$
\omega=\left(x_{1}+1\right) \sin ^{2} h_{1} D \Pi .
$$

The differential equation for the stress function $\Pi$ we obtain after substituting (22) - (24) (17):

$$
\begin{aligned}
D D^{4} \sin ^{2} h_{1} D & +2 \mu_{1} x_{1} D^{2}\left(1-0,1 h^{2} \frac{2-v}{1-v} D^{2}\right)\left(1+\frac{\sin 2 h_{1} D}{2 h_{1} D}\right) \Pi= \\
& =\left(1-\frac{h^{2}}{60} \frac{12-v}{1-v} D^{2}\right) q(x, y) .
\end{aligned}
$$

Where

$$
q(x, y)=\lambda_{1} x_{1}\left[\Phi_{y y} \sin ^{2} h_{1} D \partial_{1}^{2} \Pi+\Phi_{, x y} \sin ^{2} h_{1} D \partial_{2}^{2} \Pi-2 \Phi_{, x y} \sin ^{2} h_{1} A D \partial_{1} \partial_{2} \Pi\right]
$$

and biharmonic function - the solution of the problem of plane stress-strain state of the plate $[1,12]$. As an example, consider the case of a uniform compression plate. Get

$$
\Phi_{, x x}=\Phi_{, y y}=-\frac{P}{h}, \quad \Phi_{x y}=0
$$

Equations (25) and expression (26) take the form

$$
\begin{gathered}
D D^{i} \sin ^{2} h_{1} D \Pi+2 \mu_{1} x_{1} D^{2}\left(1-\frac{h^{2}}{10} \frac{2-v}{1-v} D^{2}\right)\left(1+\frac{\sin 2 h_{1} D}{2 h_{1} D}\right) \Pi+ \\
+\lambda\left(1+x_{1}\right) \frac{P}{h}\left(1-\frac{h^{2}}{60} \frac{12-v}{1-v} D^{2}\right) D^{2} \sin ^{2} h_{1} D \Pi=0 \\
q(x, y)=-\lambda\left(1+x_{1}\right) \frac{P}{h} D^{2} \sin ^{2} h_{1} D \Pi
\end{gathered}
$$

In order to solve a wide range of engineering problems associated with the problem of buckling of rectangular plates of different purposes, is often used the method by which the plate is given some form of depression. For hinged plates such form is received in the form of

$$
\omega=a \sin \alpha x \sin \beta y,
$$

and stresses the function $\Pi$ is determined by the differential equation (24):

$$
\left(x_{1}+1\right) \sin ^{2} h_{1} D \Pi=a \sin \alpha x \sin \beta y .
$$


A particular solution of this equation has the form $[2,13]$

$$
\Pi=\frac{a}{\left(x_{1}+1\right) \sin ^{2} i h_{1} \gamma} \sin \alpha x \sin \beta y,
$$

where $\gamma^{2}=\alpha^{2}+\beta^{2}$.

After substituting (31) into (28) we obtain the equation for $\lambda$ :

$$
2 D \gamma^{2}-\frac{2 \mu_{1} x_{1} h_{1}}{\sin ^{2} i h_{1} \gamma}\left(1-0,1 h^{2} \gamma^{2} \frac{2-v}{1-v}\right)\left(1+\frac{\sin ^{2} i h_{1} \gamma}{2 i h_{1} \gamma}\right)-\lambda\left(1+\frac{h^{2}}{60} \frac{12-v}{1-v} D^{2} \gamma^{2}\right)=0
$$

The lowest value $\lambda$ characterizes the magnitude of the load on the contour $\Gamma$, at which is fixed the loss of stability $[14,15]$.

\section{Results and discussions}

Applied theories

1. If E. Reysner plate is simply supported along the contour $\mathrm{T}$, the equilibrium equation of forms can be obtained at $\mu_{1} \rightarrow 0$ and $x_{1} \rightarrow 0$. From (4) and (16) we obtain

$$
D D^{4} \omega=\lambda\left(1-\frac{h^{2}}{60} \frac{12-v}{1-v} D^{2}\right)\left(\omega_{x x} \Phi_{y y}+\omega_{y y} \Phi_{x x}-2 \omega_{x y} \Phi_{x y}\right)
$$

Power characteristics are determined by formulas (5) - (8). Neglecting the term with $h_{2}$, we get the results of the classical theory.

2. If the expansions of differential operators retains members with $h_{1}^{2}$, we get the base model of Winkler. In the case of the equation of equilibrium forms a uniform compression plate as follows:

$$
\begin{gathered}
D D^{4} \Pi+2 \frac{\mu_{1} x_{1}}{h_{1}} \Pi+\frac{1+x_{1}}{2} \lambda \frac{P}{h} D^{2} \Pi=0 ; \\
\omega=\left(1+x_{1}\right) \Pi
\end{gathered}
$$

As an example, consider the size of the plate buckling $\mathrm{a} \times \mathrm{b}$. Assume that the deflection plates and a function of stress is expressed as

$$
\omega=\left(1+x_{1}\right) A \sin \frac{\pi x}{a} \sin \frac{\pi y}{b}, \quad \Pi=A \sin \frac{\pi x}{a} \sin \frac{\pi y}{b},
$$

Substituting (36) in (34), define the parameter $\lambda$ buckling under consideration:

$$
D\left[\left(\frac{\pi}{a}\right)^{2}+\left(\frac{\pi}{b}\right)^{2}\right]+2 \frac{\mu_{1} x_{1}}{h_{1}}-\frac{1+x_{1}}{2} \lambda \frac{P}{h}\left[\left(\frac{\pi}{a}\right)^{2}+\left(\frac{\pi}{b}\right)^{2}\right]=0 .
$$

Ratios between the size of the plate are installed using the equilibrium conditions 


$$
\iint_{S} \sigma_{z} d x d y-2\left[\int_{0}^{b} Q_{x} d y+\int_{0}^{a} Q_{y} d x\right]_{\Gamma}=0 .
$$

Because the shear forces $\left.Q_{x}\right|_{\Gamma},\left.Q_{y}\right|_{\Gamma}$ and contact stress $\left.\sigma_{z}\right|_{z=h / 2}$ have the form

$$
\begin{aligned}
& \left.Q_{x}\right|_{x=0}=2 D \frac{\pi}{\alpha}\left[\left(\frac{\pi}{a}\right)^{2}+\left(\frac{\pi}{b}\right)^{2}\right] A \sin \frac{\pi y}{b} ; \\
& \left.Q_{g}\right|_{g=0}=2 D \frac{\pi}{\alpha}\left[\left(\frac{\pi}{a}\right)^{2}+\left(\frac{\pi}{b}\right)^{2}\right] A \sin \frac{\pi x}{a} ; \\
& \left.\sigma_{z}\right|_{z=h / 2}=-L_{1} \frac{\mu_{1} x_{1}}{h_{1}} A \sin \frac{\pi x}{a} \sin \frac{\pi y}{b},
\end{aligned}
$$

then, after integration (38), we obtain

$$
\frac{1}{a^{2}}+\frac{1}{b^{2}}=\frac{1}{\pi^{2}} \sqrt{\frac{2 \mu_{1} x_{1}}{h_{1} D}} .
$$

From (37) and (41) one can get a simpler Equation:

$$
\lambda=\frac{8 \mu_{1} x_{1}}{h_{1} \pi^{2}}\left(\frac{P}{h} \frac{a^{2}+b^{2}}{a^{2} b^{2}}\right)^{-1} .
$$

\section{Conclusion}

Thus, E. Reysner theory in the zero approximation leads to results of the classical theory. At the same time fulfill all the plate boundary conditions of equilibrium. This is an advantage of the method.

\section{References}

1. S.P. Timoshenko, S. Voynovskiy, Krieger-plates and shells (Fizmatgiz, Moscow, 1963)

2. A.I. Lurie, Spatial problems of the theory of elasticity (GITTL, Moscow, 1955)

3. C.P.Wu, W.Lai, Composite Structures 122, 390-404 (2015)

4. J. Wang, X. Wang, M.Huang, International Journal of Solids and Structures 29(10), 2331239 (1992)

5. V.J. Karam, J.C.F. Telles, Engineering Analysis with Boundary Elements 9(4), 351-357 (1992)

6. L. Xiao-Yan, Computers \& Structures 54(6), 1085-1090 (1995)

7. C. Chinosi, M. Cinefra, L.D. Croce, E. Carrera, Composite Structures 99, 443-452 (2013)

8. S. Brischetto, E. Carrera, Computers \& Structures 88(23-24), 1474-1483 (2010) 
9. W.L.A. Pereira, V.J. Karam, J.A.M. Carrer, W.J. Mansur, Engineering Analysis with Boundary Elements 36(7), 1138-1150, (2012)

10. S.S. Atteshamuddin, M.G. Yuwaraj, Composite Structures 129, 177-201 (2015)

11. V.L. Berdichevsky, International Journal of Engineering Science 48(3), 383-404 (2010)

12. S.S. Phoenix, B.N. Singh, S.K. Satsangi, Composite Structures 93(2), 590-598 (2011)

13. L. Iurlaro, M. Gherlone, M. Di Sciuva, A. Tessler, Composite Structures 133, 809-817 (2015)

14. G.R. Fernandes, D.H. Konda, Engineering Analysis with Boundary Elements 36(9), 1377-1388 (2012).

15. B. Litewka, R. Sygulski, Engineering Analysis with Boundary Elements 34(12), 10721081 (2010) 